\title{
A Novel Approach on the Joint De-Identification of Textual and Relational Data with a Modified Mondrian Algorithm
}

\author{
F. Singhofer \\ fabian.singhofer@uni-ulm.de \\ University of Ulm \\ Germany
}

\author{
A. Garifullina, M. Kern \\ \{aygul.garifullina,mathias.kern\}@bt.com \\ BT \\ United Kingdom
}

\author{
A. Scherp \\ ansgar.scherp@uni-ulm.de \\ University of Ulm \\ Germany
}

\begin{abstract}
Traditional approaches for data anonymization consider relational data and textual data independently. We propose rx-anon, an anonymization approach for heterogeneous semi-structured documents composed of relational and textual attributes. We map sensitive terms extracted from the text to the structured data. This allows us to use concepts like $k$-anonymity to generate a joint, privacypreserved version of the heterogeneous data input. We introduce the concept of redundant sensitive information to consistently anonymize the heterogeneous data. To control the influence of anonymization over unstructured textual data versus structured data attributes, we introduce a modified, parameterized Mondrian algorithm. We evaluate our approach with two real-world datasets using a Normalized Certainty Penalty score, adapted to the problem of jointly anonymizing relational and textual data. The results show that our approach is capable of reducing information loss by using the tuning parameter to control the Mondrian partitioning while guaranteeing $k$-anonymity. As rx-anon is a framework approach, it can be reused and extended by other anonymization algorithms, privacy models, and textual similarity metrics.
\end{abstract}

\section{CCS CONCEPTS}

- Security and privacy $\rightarrow$ Data anonymization and sanitization; • Computing methodologies $\rightarrow$ Information extraction.

\section{KEYWORDS}

data anonymization, heterogeneous data, k-anonymity

\section{ACM Reference Format:}

F. Singhofer, A. Garifullina, M. Kern, and A. Scherp. 2021. A Novel Approach on the Joint De-Identification of Textual and Relational Data with a Modified Mondrian Algorithm. In ACM Symposium on Document Engineering 2021 (DocEng '21), August 24-27, 2021, Limerick, Ireland. ACM, New York, NY, USA, 18 pages. https://doi.org/10.1145/3469096.3469871

Permission to make digital or hard copies of all or part of this work for personal or classroom use is granted without fee provided that copies are not made or distributed for profit or commercial advantage and that copies bear this notice and the full citation on the first page. Copyrights for components of this work owned by others than the author(s) must be honored. Abstracting with credit is permitted. To copy otherwise, or republish, to post on servers or to redistribute to lists, requires prior specific permission and/or a fee. Request permissions from permissions@acm.org.

DocEng '21, August 24-27, 2021, Limerick, Ireland

(c) 2021 Copyright held by the owner/author(s). Publication rights licensed to ACM ACM ISBN 978-1-4503-8596-1/21/08 . .\$15.00

https://doi.org/10.1145/3469096.3469871

\section{Supplementary Materials}

\section{A EXTENDED EXPERIMENTAL RESULTS}

The following sections contain extended experiment results. In particular, we provide numbers of distinct entities, give information about the performance of our framework, share statistics relevant for partitioning, and present details on information loss.

\section{A.1 Distinct Terms}

Table 1 provides an overview of the number of distinct terms appearing in textual attributes. In general, the texts of the Blog Authorship Corpus contain significantly more distinct entities.

Table 1: Numbers of distinct terms per entity type. The Blog Authorship Corpus contains one textual attribute text. The Hotel Reviews Dataset contains two textual attributes, namely negative review and positive review.

\begin{tabular}{l|r|r|r}
\hline & $\begin{array}{c}\text { Blog Au- } \\
\text { thorship } \\
\text { Corpus }\end{array}$ & \multicolumn{2}{|c}{$\begin{array}{c}\text { Hotel Reviews } \\
\text { Dataset }\end{array}$} \\
entity type & \multicolumn{1}{|c}{$\begin{array}{c}\text { text } \\
\text { negative } \\
\text { review }\end{array}$} & $\begin{array}{c}\text { positive } \\
\text { review }\end{array}$ \\
\hline DATE & 83,972 & 2,672 & 1,993 \\
EVENT & 13,883 & 161 & 244 \\
FAC & 32,864 & 3,452 & 14,070 \\
GPE & 34,639 & 1,058 & 2,512 \\
LANGUAGE & 761 & 30 & 30 \\
LAW & 5,153 & 12 & 3 \\
LOC & 13,635 & 480 & 1,370 \\
MAIL & 3,225 & 0 & 0 \\
MONEY & 16,050 & 2,089 & 625 \\
NORP & 9,676 & 293 & 344 \\
ORG & 162,555 & 3,887 & 9,444 \\
PERCENT & 4,104 & 30 & 25 \\
PERSON & 245,667 & 2,273 & 5,728 \\
PHONE & 442 & 1 & 0 \\
POSTCODE & 739 & 7 & 8 \\
PRODUCT & 48,207 & 842 & 892 \\
TIME & 61,669 & 4,311 & 2,366 \\
URL & 29,297 & 0 & 0 \\
WORK_OF_ART & 145,421 & 290 & 349 \\
\hline & & &
\end{tabular}




\section{A.2 Performance}

Table 2 provides valuable insights in execution times of the experiments. Each experiment was executed on a single CPU core and did not require to analyze the texts, since the processed Natural Language Processing (NLP) state is read from cached results. In the case of experiments run on the Blog Authorship Corpus, execution times were significantly higher compared to the Hotel Reviews dataset. One observation is that if only relational attributes are considered (Mondrian, $\lambda=1$ ), execution times come down to a fraction of experiments where sensitive terms are considered during the partitioning phase.

Considering memory consumption, running a single experiment on the Blog Authorship Corpus required 25.2 GB for all entities and 13.4 GB in the case of just considering GPE entities (locations). In the case of the Hotel Review dataset, 5.4 GB and 4.2 GB were required respectively.

Table 2: Execution times of experiments in hh:mm:ss.

\begin{tabular}{|c|c|c|c|c|c|}
\hline & \multirow[b]{2}{*}{$\lambda$} & \multicolumn{2}{|c|}{$\begin{array}{l}\text { Blog Authorship } \\
\text { Corpus }\end{array}$} & \multicolumn{2}{|c|}{$\begin{array}{c}\text { Hotel Reviews } \\
\text { Dataset }\end{array}$} \\
\hline & & all & GPE & all & GPE \\
\hline 㭊 & - & $10: 25: 12$ & 03:50:13 & $00: 12: 55$ & $00: 10: 26$ \\
\hline \multirow{11}{*}{ 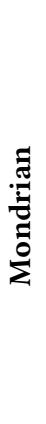 } & 0 & $15: 19: 31$ & 02:46:14 & $00: 29: 26$ & $00: 11: 44$ \\
\hline & 0.1 & $15: 01: 34$ & $01: 32: 25$ & $00: 29: 56$ & $00: 13: 16$ \\
\hline & 0.2 & $14: 43: 37$ & 01:30:11 & $00: 29: 44$ & $00: 12: 58$ \\
\hline & 0.3 & $14: 32: 23$ & 01:24:31 & $00: 29: 17$ & $00: 12: 49$ \\
\hline & 0.4 & 13:59:02 & $01: 15: 58$ & $00: 27: 59$ & $00: 12: 44$ \\
\hline & 0.5 & $11: 03: 43$ & 01:04:59 & $00: 25: 13$ & $00: 12: 18$ \\
\hline & 0.6 & $11: 05: 29$ & 01:05:04 & $00: 25: 12$ & $00: 12: 20$ \\
\hline & 0.7 & $11: 06: 26$ & 01:04:47 & $00: 25: 14$ & $00: 12: 20$ \\
\hline & 0.8 & $11: 03: 33$ & 01:04:50 & $00: 25: 01$ & $00: 12: 23$ \\
\hline & 0.9 & $11: 02: 57$ & 01:04:32 & $00: 25: 12$ & $00: 12: 19$ \\
\hline & 1 & 01:48:41 & $00: 47: 22$ & $00: 12: 48$ & $00: 11: 17$ \\
\hline
\end{tabular}

\section{A.3 Partitions}

In our experiments, we evaluate statistics on partition splits to gain insights how $\lambda$ influences splitting decisions of Mondrian partitioning. Moreover, we also share statistics on resulting partitions.

A.3.1 Partition Splits. Figure 1 provides an overview of the distribution of splitting decisions between relational and textual attributes for experiments run on the Blog Authorship Corpus for all values of $k$. The left column includes experiments considering all entities, while the right column presents results for experiments run only considering location (GPE) entities.

A noteworthy observation is that for a fixed $\lambda$, the number of splits on textual attributes decreases if $k$ increases. Since we are only considering valid splits, sensitive terms have to appear at least $2 k$ times within a partition to be split on. Therefore, in case of $k=50$, sensitive terms are required to appear 100 times, which is less likely due to heterogeneity of blog post texts.
If only locations, i. e., entities of type GPE, are considered, $\lambda$ is not in all cases able to control the share of splits between relational and textual attributes, since low values for $\lambda$ do not result in more splits on textual attributes. This effect is caused by the lack of multi-dimensionality. Since only one category of sensitive entity types is considered, Mondrian has only one option (namely split on sensitive terms with type GPE) to split on textual attributes. If splits on GPE terms fail (e. g., if there are none), Mondrian will ultimately continue to split on a relational attribute.

Similarly, Figure 2 highlights the impact of $\lambda$ on partition splits for experiments run on the Hotel Reviews Dataset for all values of $k$.

A.3.2 Partition Count and Size. We present the results regarding partition statistics. Table 3 provides valuable insights on the number of partitions as well as the size and standard deviation regarding partition sizes for the experiments on the Blog Authorship Corpus considering all entities. Similarly, Table 4 provides an overview of the same metrics for the Blog Authorship Corpus only considering GPE entities (locations). Tables 5 and 6 share insights on partition statistics for the Hotel Reviews dataset.

\section{A.4 Information Loss}

In addition to evaluating resulting partitions, we are also interested in the actual information loss which is introduced by anonymizing a given dataset. Figure 3 provides an overview of information loss for experiments run on the Blog Authorship Corpus. In particular, Figure $3 \mathrm{a}$ visualizes relational and Figure $3 \mathrm{~b}$ textual information loss for experiments considering all entity types. Similarly, Figure $3 \mathrm{c}$ and Figure $3 \mathrm{~d}$ provide an overview of information loss if only GPE entities are considered. Figure 6 provides the same statistics for the Hotel Reviews Dataset.

Moreover, Figures 4 and 5 provide a zoomed version of Figure $3 \mathrm{~b}$ and Figure $3 \mathrm{~d}$ showing textual information loss for experiments on the Blog Authorship Corpus. Similarly, we visualize zoomed textual information loss for the Hotel Reviews Dataset in Figure 7 and Figure 8.

In addition to high-level charts on information loss, Figure 9 provides a detailed analysis of information loss per entity type for the attribute text in the Blog Authorship Corpus. Additionally, Figure 10 and Figure 11 visualize the textual information loss per entity type for the attributes negative review and positive review respectively.

\section{B EXTENDED RELATED WORK}

We present related work for anonymization of other types of data.

\section{B.1 Research in Data Anonymization for Audio, Images, and Video}

Even though this work only focuses on structured data and free text, recent work on anonymization of other forms of data is worth mentioning. For de-identification of images showing faces, Gross et al. [4] highlighted that pixelation and blurring offers poor privacy and suggested a model-based approach to protect privacy while preserving data utility. In contrast, recent work by Hukkelås et al. [5] applied methods from machine learning by implementing 
a simple Generative Adversarial Network (GAN) to generate new faces to preserve privacy while retaining original data distribution

For audio data, recent work focused either on anonymization of the speaker's identity or the speech content. Justin et al. [6] suggested a framework which automatically transfers speech into a de-identified version using different acoustical models for recognition and synthesis. Moreover, Cohn et al. [2] investigated the task of de-identifying spoken text by first using Automatic Speech Recognition (ASR) to transcribe texts, then extracting entities using Named Entity Recognition (NER), and finally aligning text elements to the audio and suppressing audio segments which should be deidentified.

Additionally, recent work by Agrawal and Narayanan [1] showed that de-identification of people can also be applied to whole bodies within videos whereas Gafni et al. [3] focused on live de-identification of faces in video streams.

Finally, McDonald et al. [7] developed a framework for obfuscating writing styles which can be used by authors to prevent stylometry attacks to retrieve their identities. When it comes to unstructured text, their approach anonymizes writing styles in text documents by analyzing stylographic properties, determining features to be changed, ranking those features with respect to their clusters, and suggesting those changes to the user.

\section{REFERENCES}

[1] Prachi Agrawal and P. J. Narayanan. 2011. Person De-Identification in Videos. IEEE Trans. on Circuits and Systems for Video Technology 21, 3 (2011), 299-310.

[2] Ido Cohn, Itay Laish, Genady Beryozkin, Gang Li, Izhak Shafran, Idan Szpektor, Tzvika Hartman, Avinatan Hassidim, and Yossi Matias. 2019. Audio Deidentification - a New Entity Recognition Task. In Conf. of the North American Chapter of the Association for Computational Linguistics. ACL, 197-204. arXiv:1903.07037

[3] Oran Gafni, Lior Wolf, and Yaniv Taigman. 2019. Live Face De-Identification in Video. In Int. Conf. on Computer Vision (ICCV). IEEE, 9377-9386.

[4] Ralph Gross, Latanya Sweeney, F. de la Torre, and Simon Baker. 2006. Model-Based Face De-Identification. In Computer Vision and Pattern Recognition. IEEE, 161-161.

[5] Håkon Hukkelås, Rudolf Mester, and Frank Lindseth. 2019. DeepPrivacy: A Generative Adversarial Network for Face Anonymization. In Advances in Visual Computing, Vol. 11844. Springer, 565-578.

[6] Tadej Justin, Vitomir Struc, Simon Dobrisek, Bostjan Vesnicer, Ivo Ipsic, and France Mihelic. 2015. Speaker de-identification using diphone recognition and speech synthesis. In 11th IEEE Int. Conf. and Workshops on Automatic Face and Gesture Recognition. IEEE, 1-7.

[7] Andrew W. E. McDonald, Sadia Afroz, Aylin Caliskan, Ariel Stolerman, and Rachel Greenstadt. 2012. Use Fewer Instances of the Letter "i": Toward Writing Style Anonymization. In Privacy Enhancing Technologies, Vol. 7384. Springer, 299-318. 
all entities
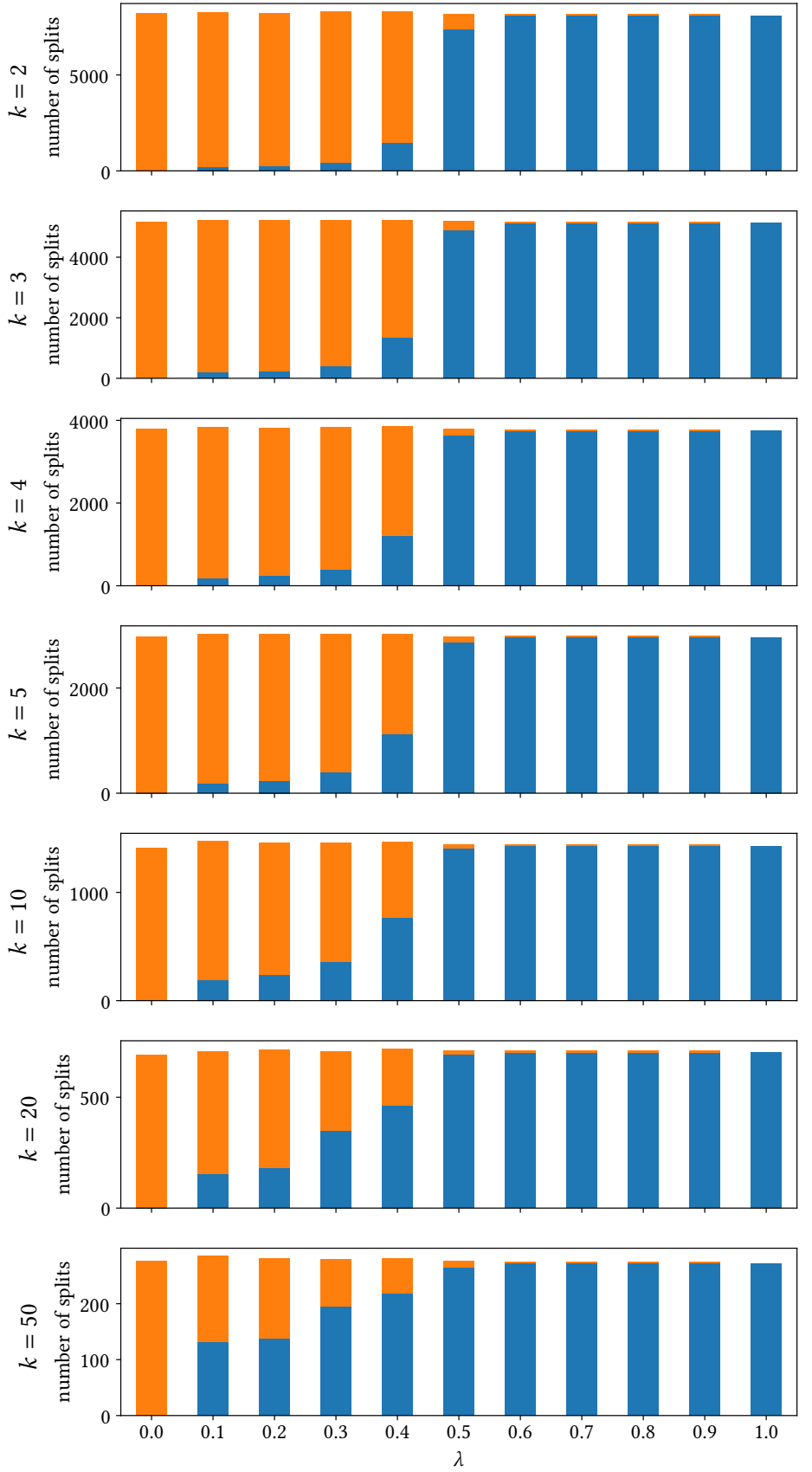

relational attribute
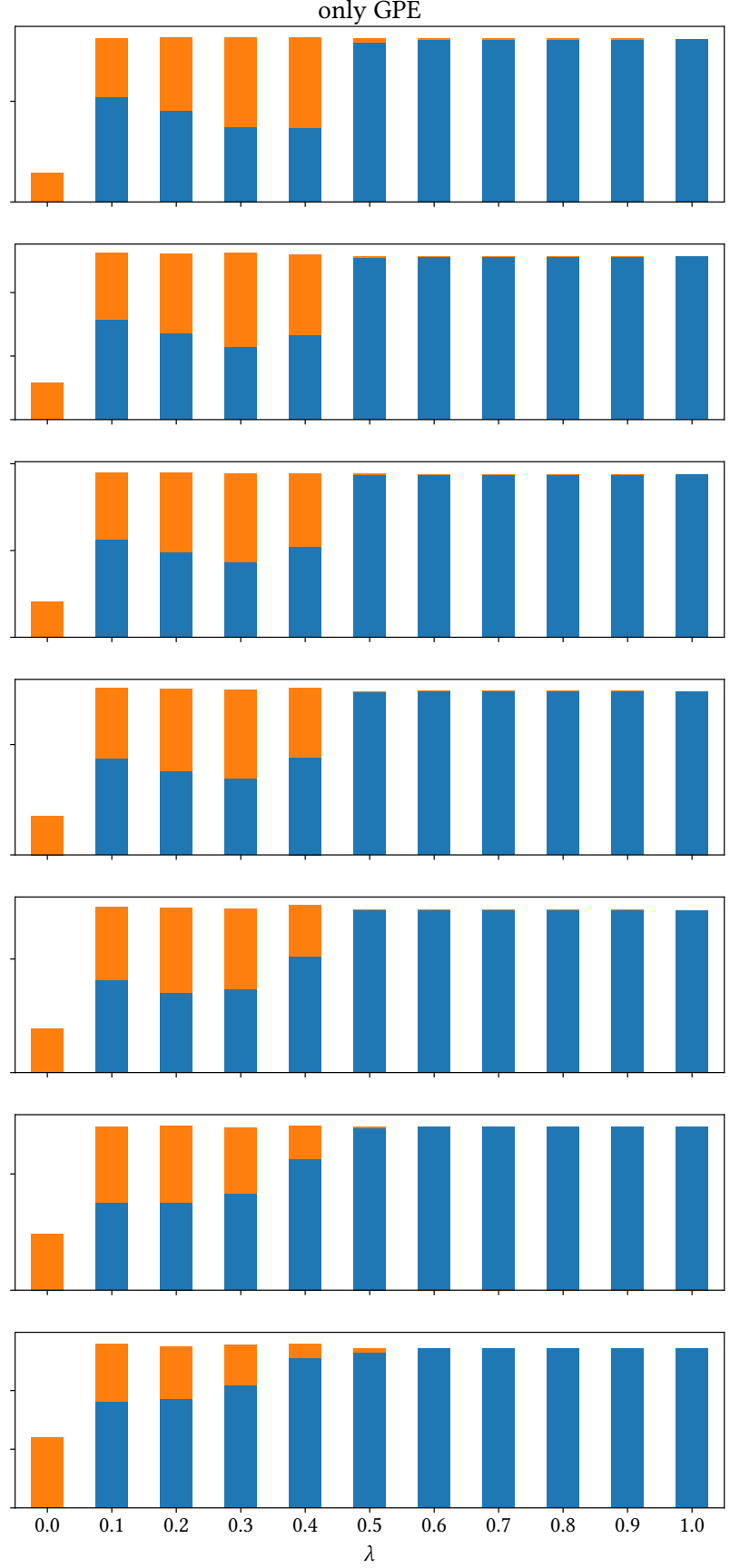

textual attribute

Figure 1: Splitting statistics for the Blog Authorship Corpus. Left plots are results for experiments run considering all entities. Right plots represent statistics for experiments run only considering GPE entities. 

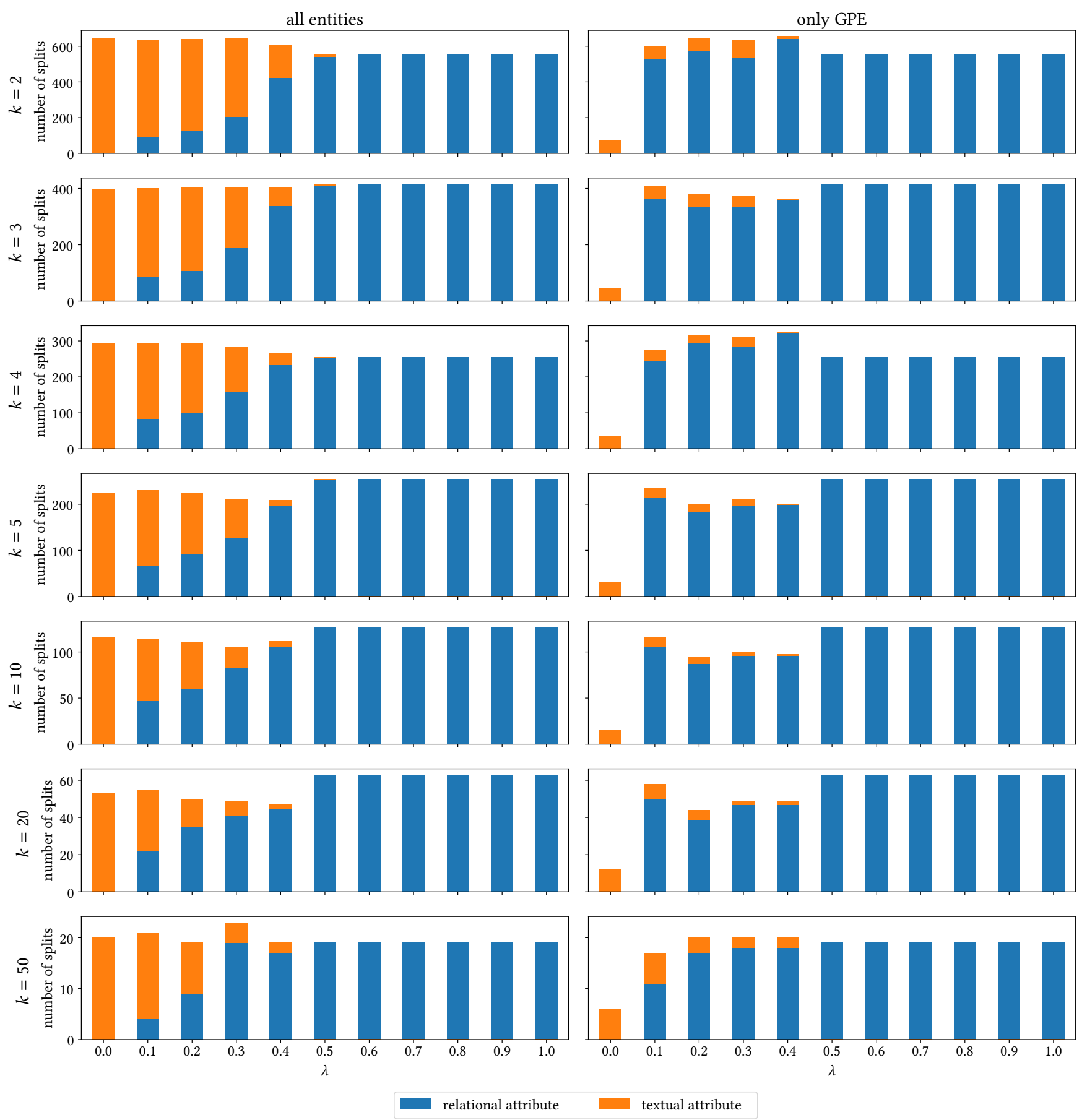

Figure 2: Splitting statistics for Hotel Reviews Dataset. Left plots are results for experiments run considering all entities. Right plots represent statistics for experiments run only considering GPE entities. 
Table 3: Statistics on resulting partitions for Blog Authorship Corpus considering all entity types.

\begin{tabular}{|c|c|c|c|c|c|c|c|c|c|}
\hline & $\lambda$ & $\mathrm{k}$ & 2 & 3 & 4 & 5 & 10 & 20 & 50 \\
\hline 家 & - & $\begin{array}{l}\text { count } \\
\text { size } \\
\text { std }\end{array}$ & $\begin{array}{r}5810 \\
3.33 \\
26.78\end{array}$ & $\begin{array}{r}2479 \\
7.79 \\
103.95\end{array}$ & $\begin{array}{r}1512 \\
12.78 \\
199.22\end{array}$ & $\begin{array}{r}1078 \\
17.92 \\
292.95\end{array}$ & $\begin{array}{r}352 \\
54.88 \\
726.15\end{array}$ & $\begin{array}{r}92 \\
209.99 \\
1742.66\end{array}$ & $\begin{array}{r}7 \\
2759.86 \\
7146.68\end{array}$ \\
\hline & 0 & $\begin{array}{l}\text { count } \\
\text { size } \\
\text { std }\end{array}$ & $\begin{array}{r}8219 \\
2.35 \\
0.76\end{array}$ & $\begin{array}{r}5162 \\
3.74 \\
1.15\end{array}$ & $\begin{array}{r}3795 \\
5.09 \\
1.48\end{array}$ & $\begin{array}{r}2971 \\
6.50 \\
1.91\end{array}$ & $\begin{array}{r}1412 \\
13.68 \\
3.56\end{array}$ & $\begin{array}{r}692 \\
27.92 \\
6.76\end{array}$ & $\begin{array}{r}278 \\
69.49 \\
18.28\end{array}$ \\
\hline & 0.1 & $\begin{array}{l}\text { count } \\
\text { size } \\
\text { std }\end{array}$ & $\begin{array}{r}8277 \\
2.33 \\
0.47\end{array}$ & $\begin{array}{r}5226 \\
3.70 \\
0.78\end{array}$ & $\begin{array}{r}3841 \\
5.03 \\
1.06\end{array}$ & $\begin{array}{r}3028 \\
6.38 \\
1.35\end{array}$ & $\begin{array}{r}1471 \\
13.13 \\
2.74\end{array}$ & $\begin{array}{r}710 \\
27.21 \\
5.77\end{array}$ & $\begin{array}{r}286 \\
67.55 \\
14.40\end{array}$ \\
\hline & 0.2 & $\begin{array}{l}\text { count } \\
\text { size } \\
\text { std }\end{array}$ & $\begin{array}{r}8243 \\
2.34 \\
0.47\end{array}$ & $\begin{array}{r}5234 \\
3.69 \\
0.79\end{array}$ & $\begin{array}{r}3829 \\
5.05 \\
1.06\end{array}$ & $\begin{array}{r}3031 \\
6.37 \\
1.36\end{array}$ & $\begin{array}{r}1460 \\
13.23 \\
2.79\end{array}$ & $\begin{array}{r}715 \\
27.02 \\
5.67\end{array}$ & $\begin{array}{r}283 \\
68.27 \\
14.64\end{array}$ \\
\hline : & 0.3 & $\begin{array}{l}\text { count } \\
\text { size } \\
\text { std }\end{array}$ & $\begin{array}{r}8302 \\
2.33 \\
0.47\end{array}$ & $\begin{array}{r}5236 \\
3.69 \\
0.78\end{array}$ & $\begin{array}{r}3841 \\
5.03 \\
1.06\end{array}$ & $\begin{array}{r}3023 \\
6.39 \\
1.34\end{array}$ & $\begin{array}{r}1462 \\
13.21 \\
2.82\end{array}$ & $\begin{array}{r}707 \\
27.33 \\
5.81\end{array}$ & $\begin{array}{r}280 \\
69.00 \\
14.47\end{array}$ \\
\hline$\sum^{0}$ & 0.4 & $\begin{array}{l}\text { count } \\
\text { size } \\
\text { std }\end{array}$ & $\begin{array}{r}8307 \\
2.33 \\
0.47\end{array}$ & $\begin{array}{r}5238 \\
3.69 \\
0.78\end{array}$ & $\begin{array}{r}3855 \\
5.01 \\
1.06\end{array}$ & $\begin{array}{r}3024 \\
6.39 \\
1.36\end{array}$ & $\begin{array}{r}1466 \\
13.18 \\
2.77\end{array}$ & $\begin{array}{r}720 \\
26.83 \\
5.57\end{array}$ & $\begin{array}{r}283 \\
68.27 \\
14.50\end{array}$ \\
\hline & 0.5 & $\begin{array}{l}\text { count } \\
\text { size } \\
\text { std }\end{array}$ & $\begin{array}{r}8186 \\
2.36 \\
0.48\end{array}$ & $\begin{array}{r}5198 \\
3.72 \\
0.77\end{array}$ & $\begin{array}{r}3800 \\
5.08 \\
1.07\end{array}$ & $\begin{array}{r}2979 \\
6.49 \\
1.34\end{array}$ & $\begin{array}{r}1441 \\
13.41 \\
2.76\end{array}$ & $\begin{array}{r}711 \\
27.17 \\
5.68\end{array}$ & $\begin{array}{r}278 \\
69.49 \\
14.59\end{array}$ \\
\hline & $0.6-0.9$ & $\begin{array}{l}\text { count } \\
\text { size } \\
\text { std }\end{array}$ & $\begin{array}{r}8168 \\
2.37 \\
0.48\end{array}$ & $\begin{array}{r}5180 \\
3.73 \\
0.77\end{array}$ & $\begin{array}{r}3781 \\
5.11 \\
1.07\end{array}$ & $\begin{array}{r}2987 \\
6.47 \\
1.35\end{array}$ & $\begin{array}{r}1441 \\
13.41 \\
2.76\end{array}$ & $\begin{array}{r}711 \\
27.17 \\
5.69\end{array}$ & $\begin{array}{r}276 \\
70.00 \\
14.43\end{array}$ \\
\hline & 1 & $\begin{array}{l}\text { count } \\
\text { size } \\
\text { std }\end{array}$ & $\begin{array}{r}8080 \\
2.39 \\
0.51\end{array}$ & $\begin{array}{r}5128 \\
3.77 \\
0.80\end{array}$ & $\begin{array}{r}3749 \\
5.15 \\
1.10\end{array}$ & $\begin{array}{r}2964 \\
6.52 \\
1.38\end{array}$ & $\begin{array}{r}1431 \\
13.50 \\
2.80\end{array}$ & $\begin{array}{r}703 \\
27.48 \\
5.78\end{array}$ & $\begin{array}{r}273 \\
70.77 \\
14.63\end{array}$ \\
\hline
\end{tabular}


Table 4: Statistics on resulting partitions for Blog Authorship Corpus considering only GPE entities

\begin{tabular}{|c|c|c|c|c|c|c|c|c|c|}
\hline & $\lambda$ & $\mathrm{k}$ & 2 & 3 & 4 & 5 & 10 & 20 & 50 \\
\hline \multirow{3}{*}{ 今) } & \multirow{3}{*}{-} & count & 2140 & 737 & 301 & 129 & 2 & 1 & 1 \\
\hline & & size & 9.03 & 26.21 & 64.18 & 149.76 & 9659.50 & 19319 & 19319 \\
\hline & & std & 575.34 & 1016.03 & 1629.78 & 13645.04 & 0 & 0 & \\
\hline \multirow{24}{*}{ 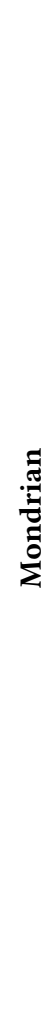 } & \multirow{3}{*}{0} & count & 1447 & 1164 & 828 & 703 & 392 & 242 & 121 \\
\hline & & size & 13.35 & 16.60 & 23.33 & 27.48 & 49.28 & 79.83 & 159.66 \\
\hline & & std & 113.84 & 126.77 & 150.14 & 162.72 & 217.34 & 275.39 & 383.25 \\
\hline & \multirow{3}{*}{0.1} & count & 8137 & 5265 & 3790 & 3019 & 1460 & 704 & 281 \\
\hline & & size & 2.37 & 3.67 & 5.10 & 6.40 & 13.23 & 27.44 & 68.75 \\
\hline & & std & 0.49 & 0.77 & 1.09 & 1.36 & 2.83 & 5.82 & 15.32 \\
\hline & \multirow{6}{*}{ 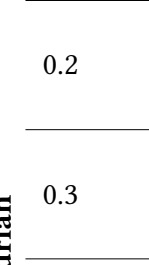 } & count & 8186 & 5227 & 3801 & 3016 & 1451 & 709 & 276 \\
\hline & & size & 2.36 & 3.70 & 5.08 & 6.41 & 13.31 & 27.25 & 70.00 \\
\hline & & std & 0.48 & 0.79 & 1.08 & 1.37 & 2.85 & 5.87 & 14.48 \\
\hline & & count & 8167 & 5248 & 3780 & 2994 & 1443 & 700 & 279 \\
\hline & & size & 2.37 & 3.68 & 5.11 & 6.45 & 13.39 & 27.60 & 69.24 \\
\hline & & std & 0.48 & 0.78 & 1.09 & 1.34 & 2.86 & 6.08 & 14.55 \\
\hline & \multirow{3}{*}{0.4} & count & 8153 & 5200 & 3780 & 3022 & 1473 & 707 & 280 \\
\hline & & size & 2.37 & 3.72 & 5.11 & 6.39 & 13.12 & 27.33 & 69.00 \\
\hline & & std & 0.48 & 0.79 & 1.08 & 1.35 & 2.74 & 5.88 & 14.07 \\
\hline & \multirow{3}{*}{0.5} & count & 8153 & 5154 & 3762 & 2970 & 1433 & 703 & 273 \\
\hline & & size & 2.37 & 3.75 & 5.14 & 6.50 & 13.48 & 27.48 & 70.77 \\
\hline & & std & 0.49 & 0.79 & 1.09 & 1.37 & 2.79 & 5.82 & 14.88 \\
\hline & \multirow{3}{*}{$0.6-0.9$} & count & 8146 & 5153 & 3761 & 2970 & 1433 & 703 & 273 \\
\hline & & size & 2.37 & 3.75 & 5.14 & 6.50 & 13.48 & 27.48 & 70.77 \\
\hline & & std & 0.49 & 0.79 & 1.09 & 1.37 & 2.79 & 5.78 & 14.63 \\
\hline & \multirow{3}{*}{1} & count & 8080 & 5128 & 3749 & 2964 & 1431 & 703 & 273 \\
\hline & & size & 2.39 & 3.77 & 5.15 & 6.52 & 13.50 & 27.48 & 70.77 \\
\hline & & std & 0.51 & 0.80 & 1.10 & 1.38 & 2.80 & 5.78 & 14.63 \\
\hline
\end{tabular}


Table 5: Statistics on resulting partitions for Hotel Reviews Dataset considering all entity types.

\begin{tabular}{|c|c|c|c|c|c|c|c|c|c|}
\hline & $\lambda$ & $\mathrm{k}$ & 2 & 3 & 4 & 5 & 10 & 20 & 50 \\
\hline \multirow{3}{*}{ (ㄴ) } & \multirow{3}{*}{-} & count & 523 & 272 & 163 & 127 & 43 & 16 & 1 \\
\hline & & size & 2.82 & 5.42 & 9.05 & 11.61 & 34.30 & 92.19 & 1475 \\
\hline & & std & 3.45 & 11.51 & 24.56 & 35.47 & 112.32 & 264.30 & 0 \\
\hline \multirow{21}{*}{ 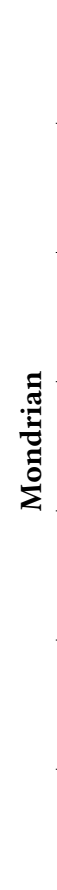 } & \multirow{3}{*}{0} & count & 645 & 398 & 293 & 226 & 117 & 54 & 21 \\
\hline & & size & 2.29 & 3.71 & 5.03 & 6.53 & 12.61 & 27.31 & 70.24 \\
\hline & & std & 0.47 & 0.77 & 1.11 & 1.45 & 2.60 & 5.59 & 15.67 \\
\hline & \multirow{3}{*}{0.1} & count & 635 & 401 & 294 & 231 & 115 & 56 & 22 \\
\hline & & size & 2.32 & 3.68 & 5.02 & 6.39 & 12.83 & 26.34 & 67.05 \\
\hline & & std & 0.47 & 0.76 & 1.04 & 1.39 & 2.78 & 5.22 & 14.78 \\
\hline & \multirow{3}{*}{0.2} & count & 641 & 404 & 295 & 224 & 112 & 51 & 20 \\
\hline & & size & 2.30 & 3.65 & 5 & 6.58 & 13.17 & 28.92 & 73.75 \\
\hline & & std & 0.46 & 0.75 & 1.08 & 1.41 & 2.71 & 5.03 & 15.00 \\
\hline & \multirow{3}{*}{0.3} & count & 645 & 404 & 285 & 212 & 106 & 50 & 24 \\
\hline & & size & 2.29 & 3.65 & 5.18 & 6.96 & 13.92 & 29.50 & 61.46 \\
\hline & & std & 0.45 & 0.79 & 1.03 & 1.41 & 2.43 & 3.65 & 6.47 \\
\hline & \multirow{3}{*}{0.4} & count & 610 & 406 & 268 & 209 & 113 & 48 & 20 \\
\hline & & size & 2.42 & 3.63 & 5.50 & 7.06 & 13.05 & 30.73 & 73.75 \\
\hline & & std & 0.49 & 0.84 & 0.85 & 1.76 & 2.32 & 6.71 & 6.45 \\
\hline & \multirow{3}{*}{0.5} & count & 558 & 415 & 256 & 255 & 128 & 64 & 20 \\
\hline & & size & 2.64 & 3.55 & 5.76 & 5.78 & 11.52 & 23.05 & 73.75 \\
\hline & & std & 0.48 & 0.84 & 0.70 & 0.72 & 1.19 & 2.22 & 19.13 \\
\hline & \multirow{3}{*}{$0.6-1$} & count & 554 & 417 & 256 & 255 & 128 & 64 & 20 \\
\hline & & size & 2.66 & 3.54 & 5.76 & 5.78 & 11.52 & 23.05 & 73.75 \\
\hline & & std & 0.47 & 0.83 & 0.70 & 0.71 & 1.19 & 2.22 & 19.13 \\
\hline
\end{tabular}


Table 6: Statistics on resulting partitions for Hotel Reviews Dataset considering only GPE entities.

\begin{tabular}{|c|c|c|c|c|c|c|c|c|c|}
\hline & $\lambda$ & $\mathrm{k}$ & 2 & 3 & 4 & 5 & 10 & 20 & 50 \\
\hline \multirow{3}{*}{ 㭊 } & \multirow{3}{*}{-} & count & 303 & 107 & 47 & 15 & 1 & 1 & 1 \\
\hline & & size & 4.87 & 13.79 & 31.38 & 98.33 & 1475 & 1475 & 1475 \\
\hline & & std & 18.59 & 90.92 & 179.98 & 358.71 & 0 & 0 & 0 \\
\hline \multirow{18}{*}{ 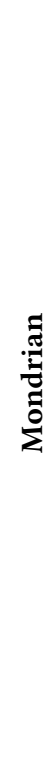 } & \multirow{3}{*}{0} & count & 74 & 49 & 35 & 32 & 17 & 13 & 7 \\
\hline & & size & 19.93 & 30.10 & 42.14 & 46.09 & 86.76 & 113.46 & 210.71 \\
\hline & & std & 61.71 & 74.77 & 86.30 & 89.59 & 112.62 & 121.77 & 135.59 \\
\hline & \multirow{3}{*}{0.1} & count & 603 & 408 & 276 & 236 & 117 & 59 & 18 \\
\hline & & size & 2.45 & 3.62 & 5.34 & 6.25 & 12.61 & 25 & 81.94 \\
\hline & & std & 0.50 & 0.72 & 1.10 & 1.06 & 1.82 & 3.03 & 13.70 \\
\hline & \multirow{3}{*}{$\begin{array}{r}0.2 \\
\end{array}$} & count & 647 & 380 & 318 & 201 & 95 & 45 & 21 \\
\hline & & size & 2.28 & 3.88 & 4.64 & 7.34 & 15.53 & 32.78 & 70.24 \\
\hline & & std & 0.45 & 0.70 & 0.89 & 1.45 & 2.70 & 4.46 & 8.09 \\
\hline & \multirow{3}{*}{0.3} & count & 634 & 376 & 312 & 211 & 101 & 50 & 21 \\
\hline & & size & 2.33 & 3.92 & 4.73 & 6.99 & 14.60 & 29.50 & 70.24 \\
\hline & & std & 0.47 & 0.75 & 0.89 & 1.55 & 2.91 & 5.75 & 7.42 \\
\hline & \multirow{3}{*}{0.4} & count & 657 & 362 & 327 & 202 & 99 & 50 & 21 \\
\hline & & size & 2.25 & 4.07 & 4.51 & 7.30 & 14.90 & 29.50 & 70.24 \\
\hline & & std & 0.43 & 0.66 & 0.75 & 1.51 & 2.82 & 5.75 & 7.42 \\
\hline & \multirow{3}{*}{$0.5-1$} & count & 554 & 417 & 256 & 255 & 128 & 64 & 20 \\
\hline & & size & 2.66 & 3.54 & 5.76 & 5.78 & 11.52 & 23.05 & 73.75 \\
\hline & & std & 0.47 & 0.83 & 0.70 & 0.71 & 1.19 & 2.22 & 19.13 \\
\hline
\end{tabular}




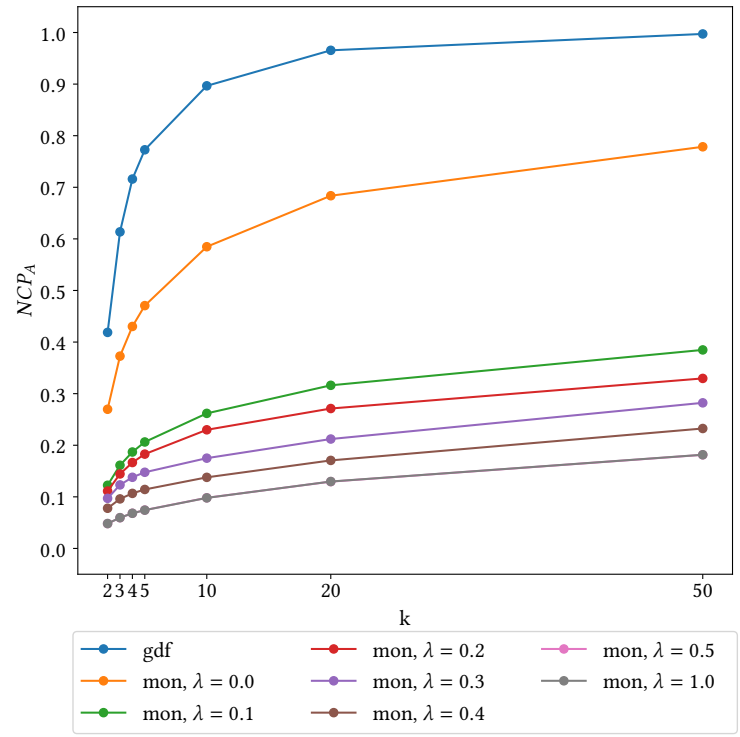

(a) Relational information loss $\mathrm{NCP}_{\mathrm{A}}$, all entity types

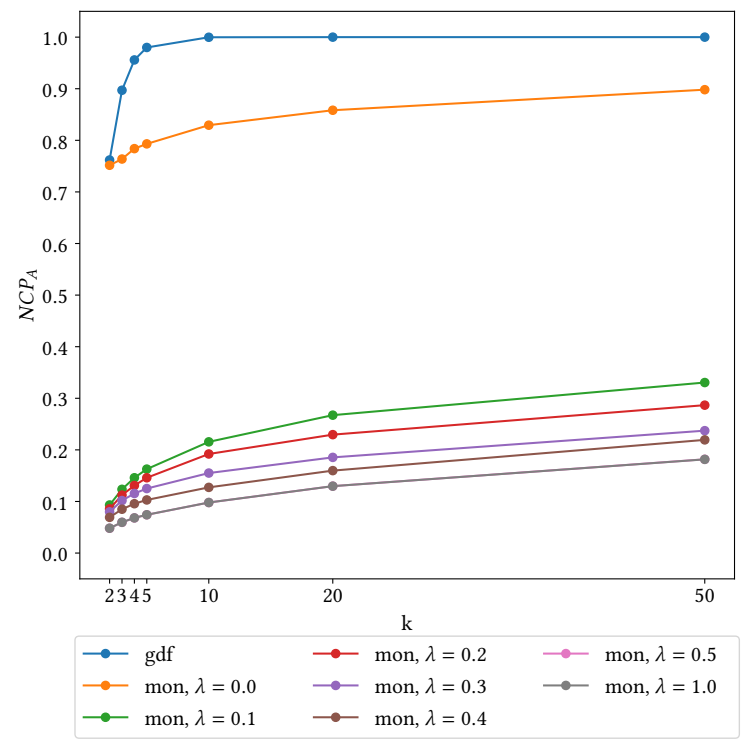

(c) Relational information loss $\mathrm{NCP}_{\mathrm{A}}$, only GPE entities

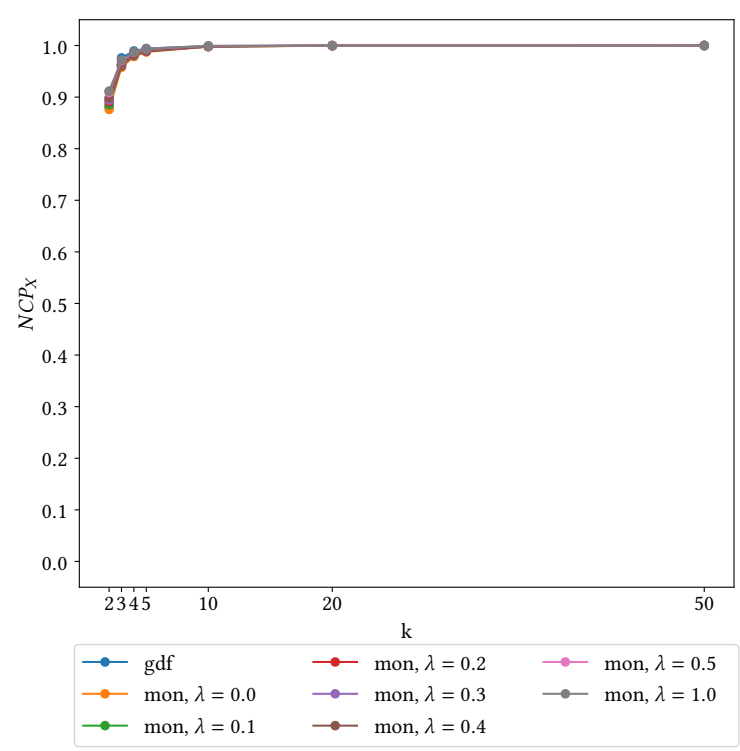

(b) Textual information loss $\mathrm{NCP}_{\mathrm{X}}$, all entity types

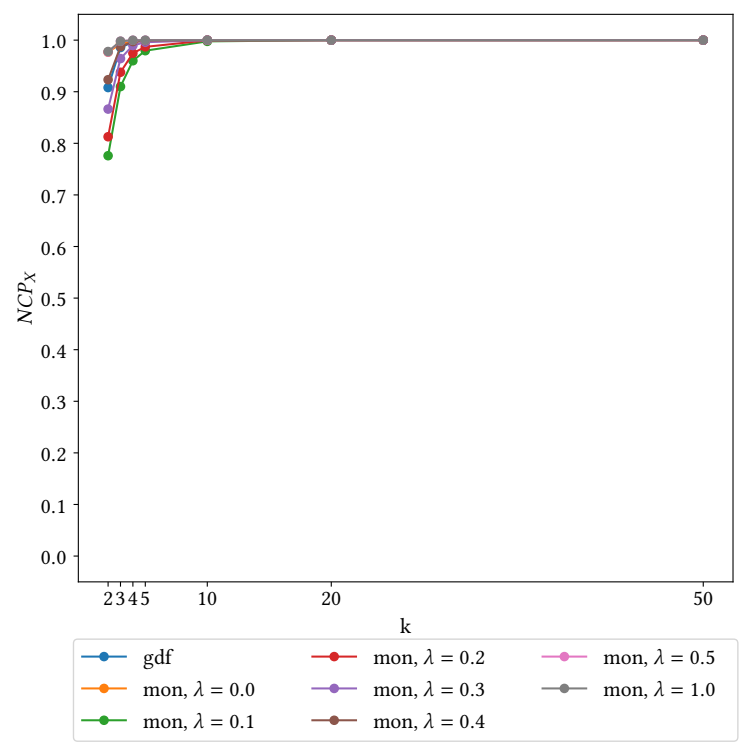

(d) Textual information loss $\mathrm{NCP}_{\mathrm{X}}$, only GPE entities

Figure 3: Relational and textual information loss for experiments run on the Blog Authorship Corpus. Results for relational (a) and textual information loss (b) for experiments considering all entities. Results for relational (c) and textual information loss (d) for experiments considering only GPE entities. 


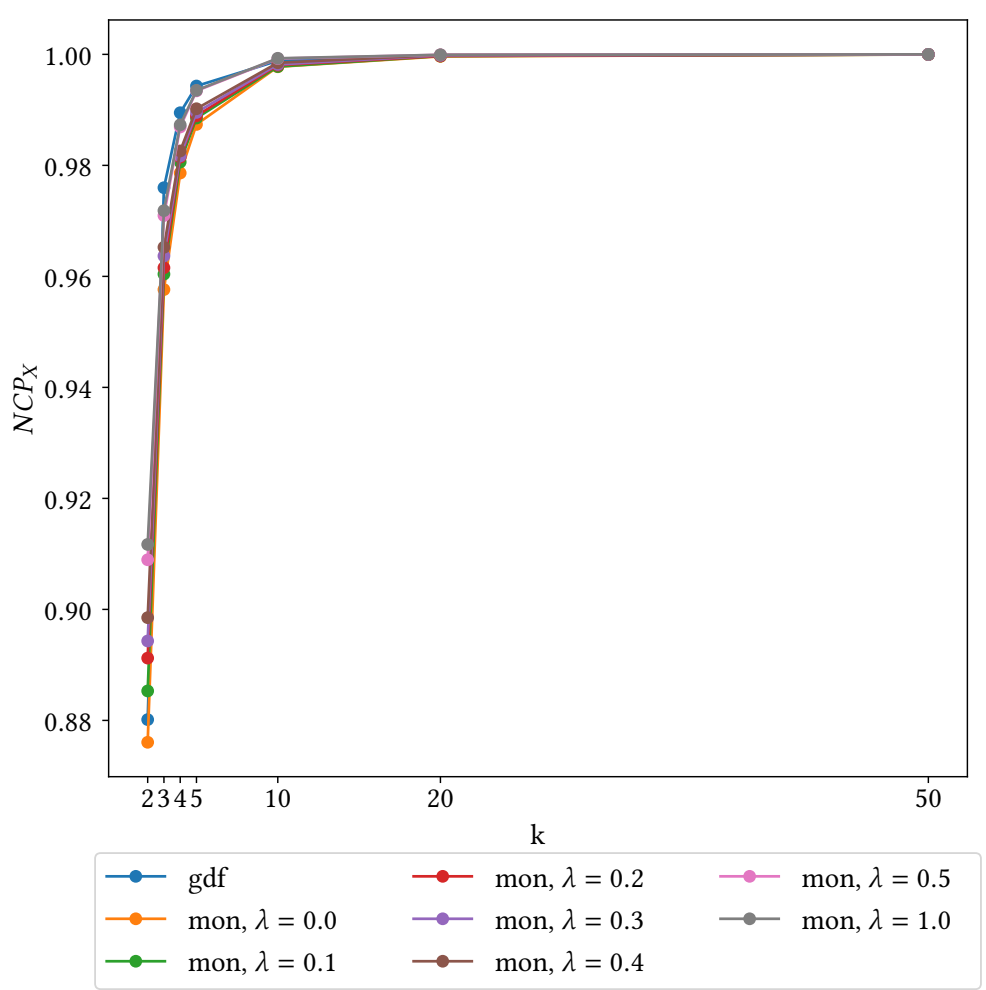

Figure 4: Zoomed textual information loss for experiments run on the Blog Authorship Corpus considering all entities. 


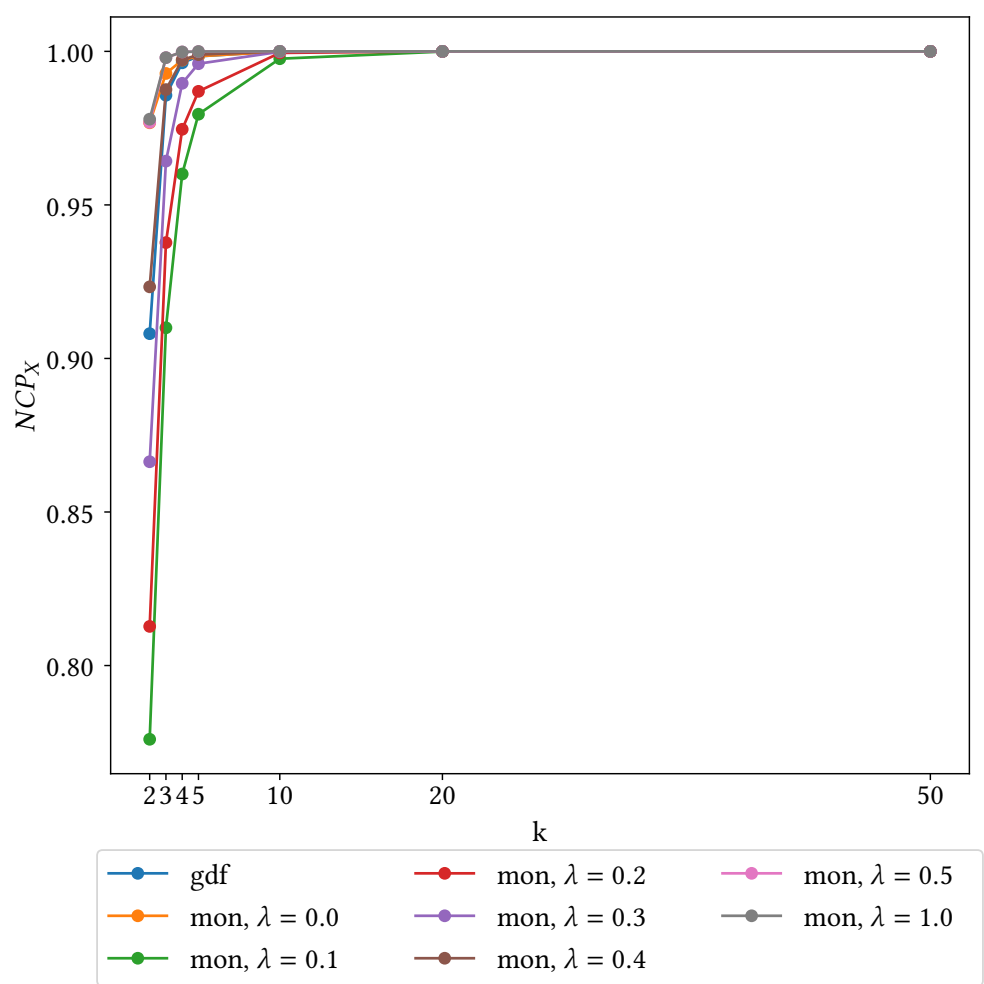

Figure 5: Zoomed textual information loss for experiments run on the Blog Authorship Corpus considering only GPE entities. 


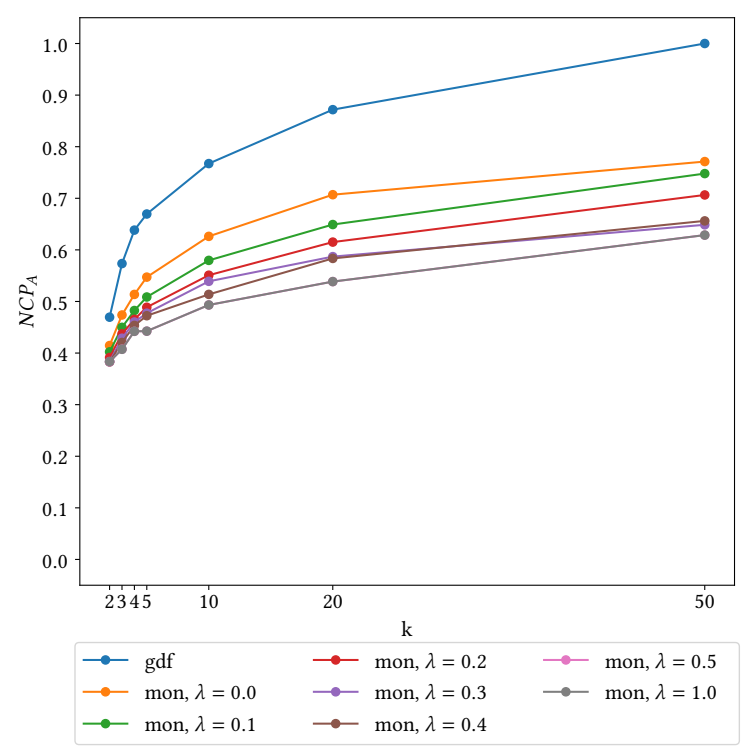

(a) Relational information loss $\mathrm{NCP}_{\mathrm{A}}$, all entity types

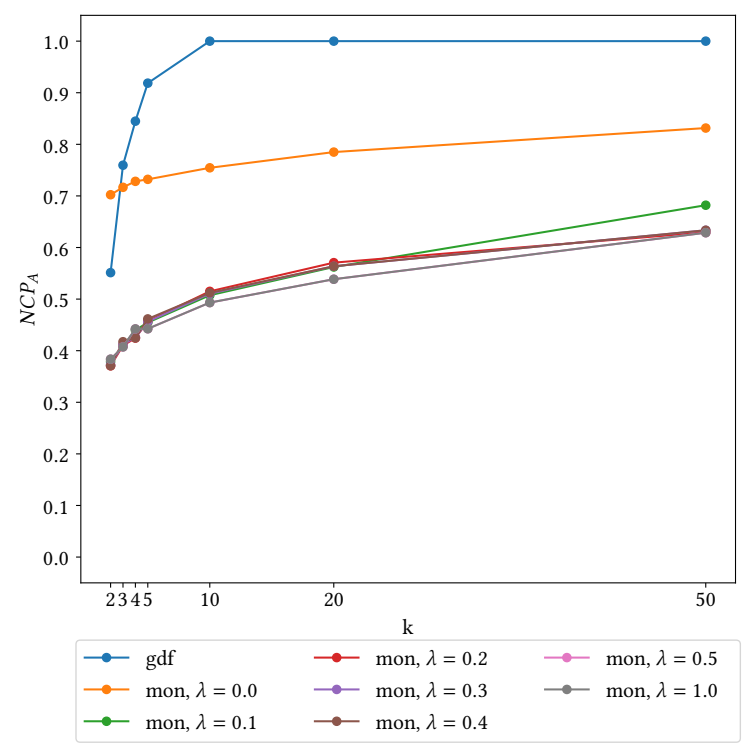

(c) Relational information loss $\mathrm{NCP}_{\mathrm{A}}$, only GPE entities

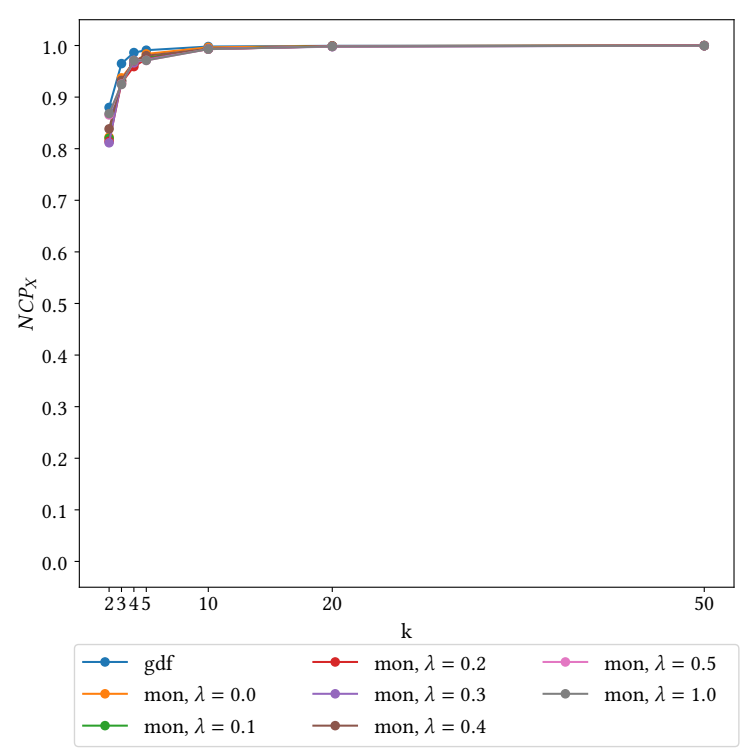

(b) Textual information loss $\mathrm{NCP}_{\mathrm{X}}$, all entity types

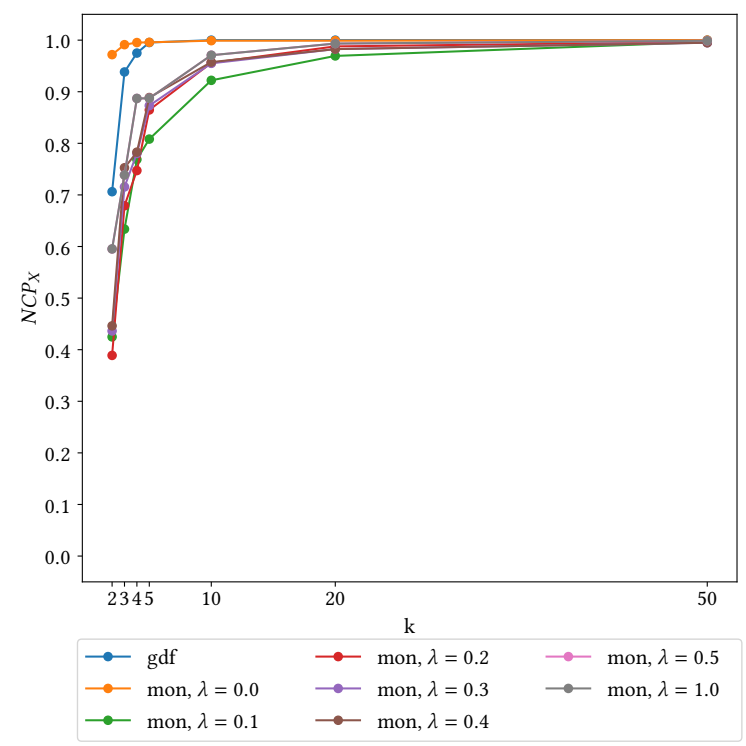

(d) Textual information loss NCPX, only GPE entities

Figure 6: Relational and textual information loss for experiments run on the Hotel Reviews Dataset. Results for relational (a) and textual information loss (b) for experiments considering all entities. Results for relational (c) and textual information loss (d) for experiments considering only GPE entities. 


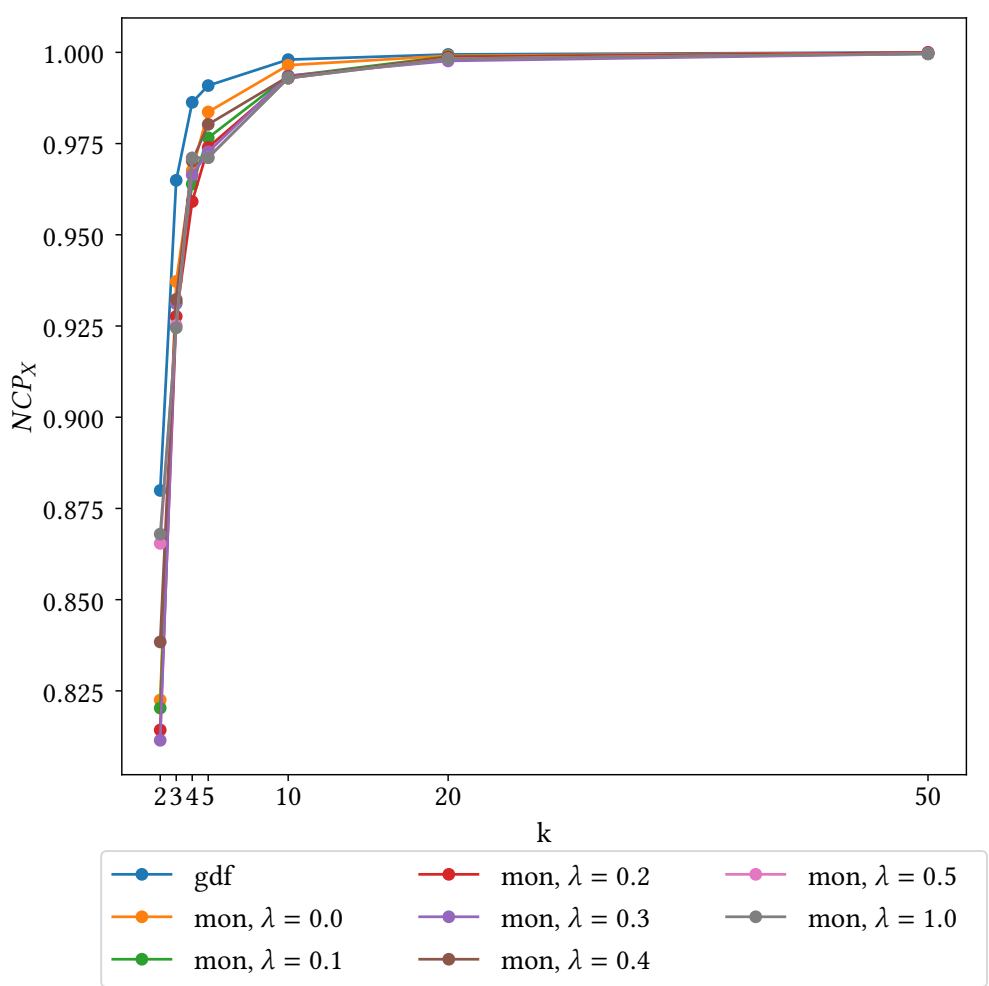

Figure 7: Zoomed textual information loss for experiments run on the Hotel Reviews Dataset considering all entities. 


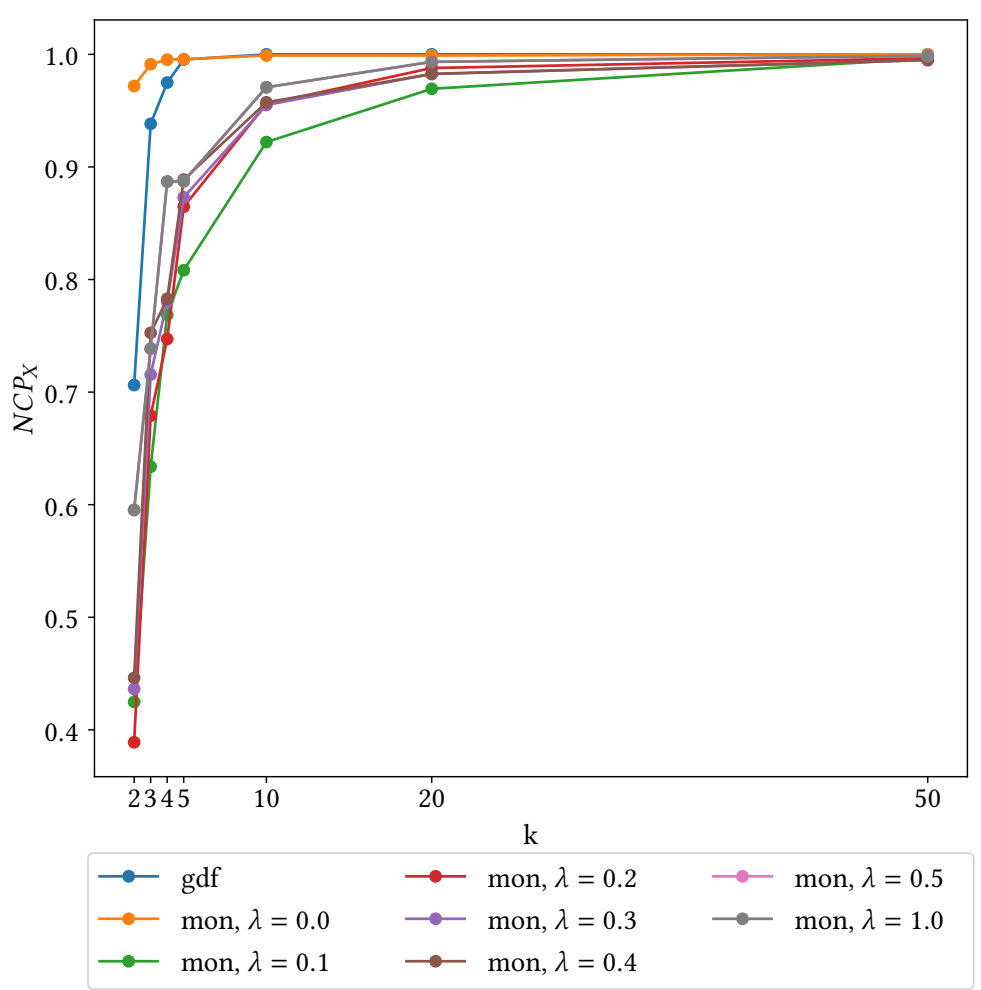

Figure 8: Zoomed textual information loss for experiments run on the Hotel Reviews Dataset considering only GPE entities. 


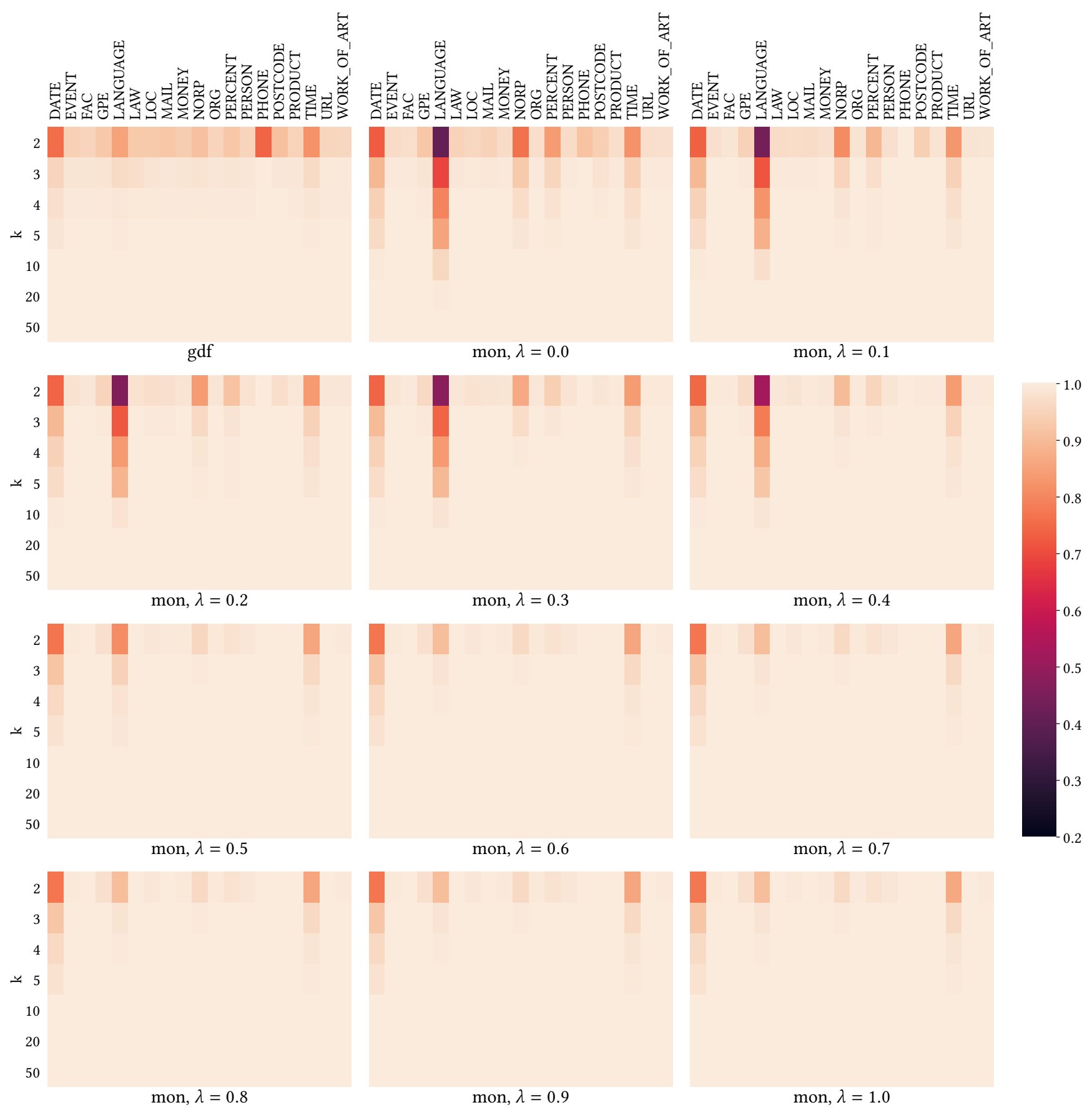

Figure 9: Textual information loss of the attribute text per entity types for experiments run on the Blog Authorship Corpus. Information loss is visualized for GDF and Mondrian partitioning with varying $\lambda$. 


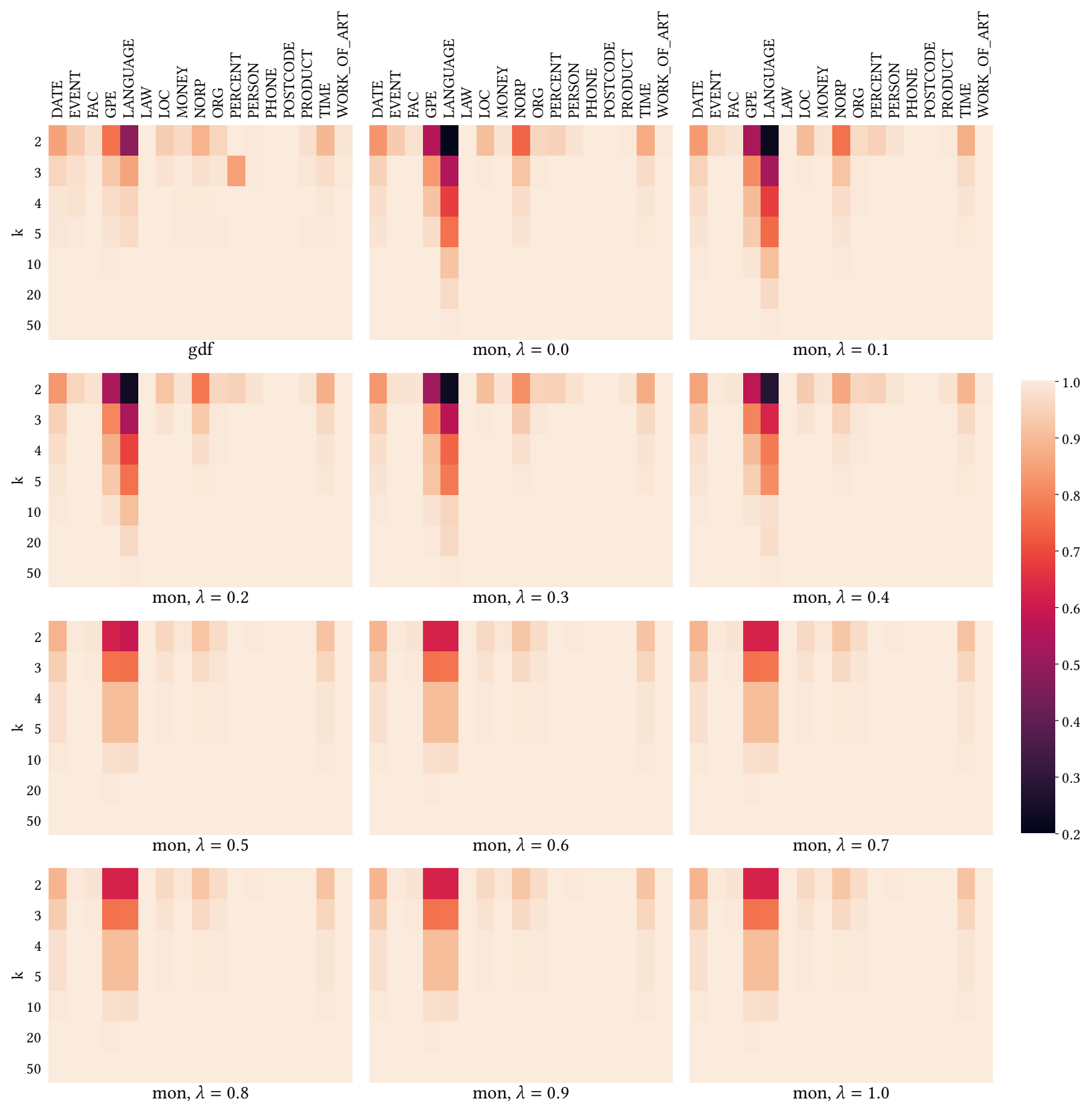

Figure 10: Textual information loss of the attribute negative review per entity type for experiments run on the Hotel Reviews Dataset. Information loss is visualized for GDF and Mondrian partitioning with varying $\lambda$. 


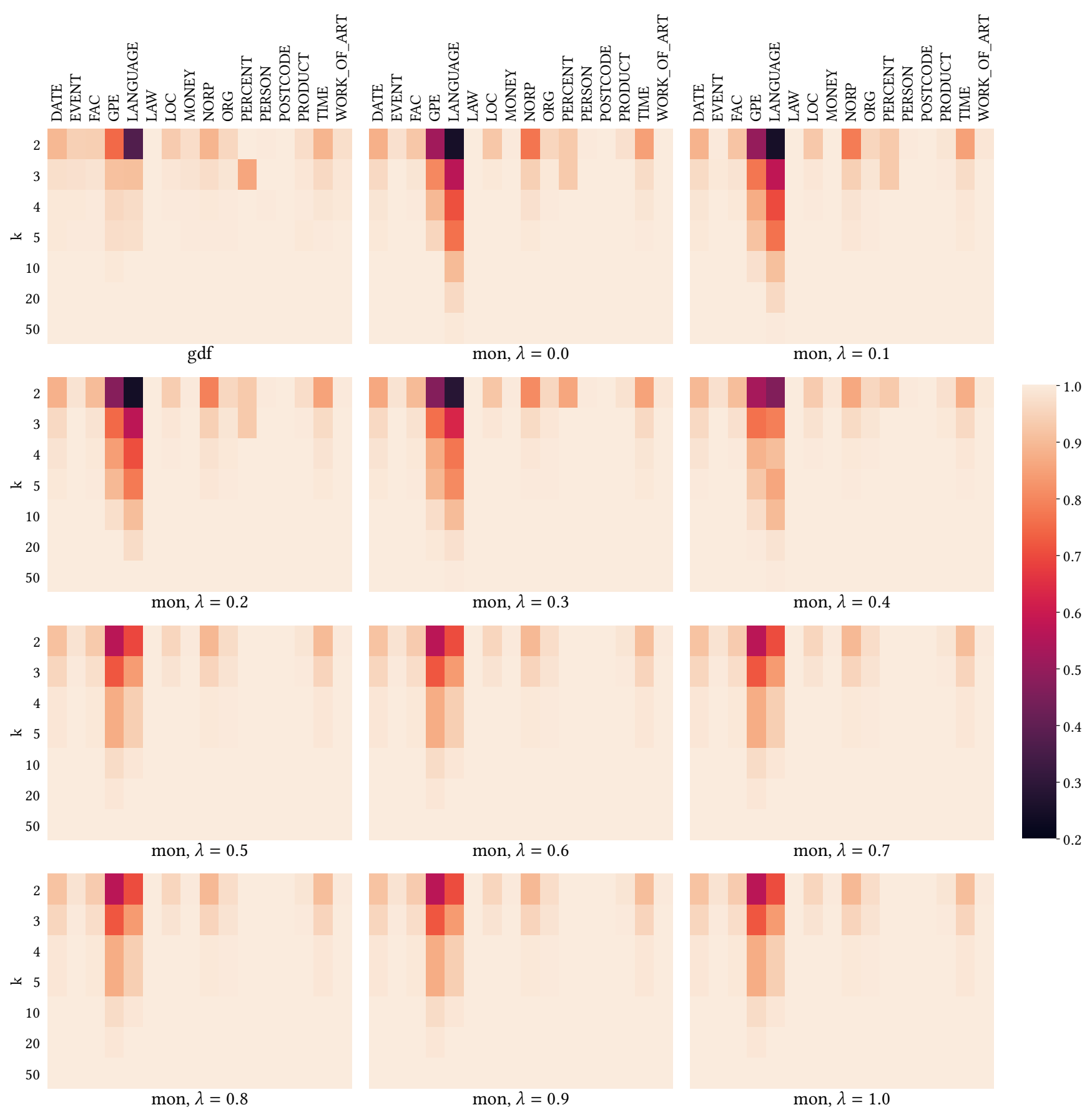

Figure 11: Textual information loss of the attribute positive review per entity type for experiments run on the Hotel Reviews Dataset. Information loss is visualized for GDF and Mondrian partitioning with varying $\lambda$. 